\title{
Applications of Structural Synthesis of Programs
}

\author{
Enn Tyugu ${ }^{1}$, Mihhail Matskin $^{2}$, and Jaan Penjam ${ }^{3}$ \\ 1 Department of Teleinformatics, Royal Institute of Technology, Kista, Sweden \\ tyugu@it.kth.se \\ 2 Department of Computer and Information Science, Norwegian University of Science \\ and Technology, Trondheim, Norway \\ Mihhail.Matskin@idi.ntnu.no \\ ${ }^{3}$ Institute of Cybernetics, Tallinn, Estonia \\ Jaan.Penjam@cs.ioc.ee
}

\begin{abstract}
This is an experience report on the automatic and hidden usage of program synthesis in several application domains. The structural synthesis of programs has been implemented in an object-oriented programming environment NUT and used for development of simulation software, engineering calculations software, implementing a benchmark for safety critical systems and development of highly interactive visual modeling of radar coverage of landscape.
\end{abstract}

\section{Introduction}

This paper presents experience of using a formal method of software development that is applied completely automatically, and hidden from the user. The advantage is that we do not introduce errors during the process of usage of a formal method in this case. The obvious disadvantages are high requirements to the reliability and performance of complex automatic operations that are hidden from the user.

Actually, there are few synthesis methods sufficiently mature for automatic usage. Here we refer to the following two practically applied systems where automatic program synthesis plays a central role: Specware [20] and Amphion [12]. Specware is a tool for creating applications from a set of specifications of its parts. The specifications are given in an algebraic style in the language called Slang. They are joined into larger specifications in a formal way specified in terms of categories. Specware includes a visual tool for drawing diagrams of categories for composing the specifications. Although Specware is intended for the interactive usage, the composition of specifications and development of an implementation is highly automated. Another automated system with deductive program synthesis is Amphion - a program for constructing programs from preprogrammed subroutines in a library. This system is domain-oriented and it is used for solving problems in planetary astronomy. Using the experience in program synthesis gained with Amphion, its authors have developed Meta-Amphion - a tool for implementing domain-oriented synthesizers [13].

We are dealing with deductive program synthesis, and are concerned not only of the synthesis process, but also of getting correct specifications as the reliable 
material for synthesis. The complete verification/validation of specifications is in principle an unsolvable task, therefore we try to give to software developers specification tools which are intuitive and easy to use. As a consequence, we have had also another and completely different motivation of our research - development of a sufficiently general method of semantic processing of specifications which is applicable in compilers of a wide class of problem-oriented languages.

The main part of this paper is a presentation of applications of program synthesis in several problem domains: design of mechanical drives, implementation of a safety-critical system, analysis of dynamics of a hydraulic system for controlling ailerons of an aircraft, calculating radar coverage of a landscape and simulation of a network of mobile telephone communication. These applications are all implemented in the NUT programming environment where the program synthesis is used hiddenly and automatically. We start with brief descriptions of the synthesis method and the programming environment.

\section{Structural Synthesis of Programs}

Structural synthesis of programs (SSP) is a deductive program synthesis method based on the idea that one can construct programs taking into account only their structural properties. We use this idea for constructing programs from small as well as large modules whose behavior we do not describe in detail. Each preprogrammed module is supplied with a specification used as an axiom stating under which conditions the module can be applied and which postconditions will be satisfied after its execution. However, the specification does not specify explicitly the relation between the input and output of the module.

The SSP uses an implicative fragment of the intuitionistic propositional calculus with restricted nestedness of implications. Intuitionistic logic guarantees simplicity of program extraction from proofs: programs are realizations of formulae and can be represented in typed lambda calculus. The general form of formulae is

$$
(\underline{A} \rightarrow B) \& \ldots \&(\underline{C} \rightarrow D) \rightarrow(\underline{E} \rightarrow F)
$$

where, for any symbol $W, \underline{W}$ denotes $W_{1} \& W_{2} \& \ldots \& W_{n}$ or $W_{1}, W_{2}, \ldots, W_{n}$ or empty (depending on context). If we assume that propositional letters $\underline{X}, Y, \ldots$ denote computability of objects $\underline{x}, y, \ldots$, then an implication $\underline{X} \rightarrow Y$ has the following meaning " $\mathrm{y}$ is computable from $\underline{x}$ ". An implication of this form can be either a specification of a preprogrammed module (i.e. an axiom), or a goal specifying the program to be synthesized. In the case of an axiom we can show the module under the arrow as follows: $\underline{X} \underset{f}{\rightarrow}$.

The nested implications $\underline{A} \rightarrow B, \ldots, \underline{C} \rightarrow D$ in an axiom of the form (1) are called subtasks. They state subgoals, which must be achieved in order to apply the module with this axiom. They provide generality to the logical language, indeed, they play a role similar to atoms in the body of a Prolog clause. 
Program development based on SSP is sometimes called propositional logic programming, because, first, it is a kind of logic programming, and, second, it uses a propositional logic [15.

The fragment of the intuitionistic propositional calculus used in SSP is still expressive enough for deductively equivalent encoding of arbitrary intuitionistic propositional formulae [15. The derivability problem in the intuitionistic propositional calculus is known to be PSPACE complete, consequently, the proof search in SSP is PSPACE complete. Still, efficient algorithms exist for practical cases of synthesis where restrictions on structural complexity of specifications are known [18].

A natural way to extend the SSP could be to use a more general theory of constructive types, e.g. proposed by P. Martin Löf [5]. However, we have been afraid of loosing the efficiency by introducing this extension.

\section{NUT Programming Environment}

The NUT programming environment is an Unix/Linux-based tool that combines object-oriented and visual programming paradigms with automatic program synthesis. The object-oriented concept of a class is extended in such a way that a class can serve as a complete specification for program synthesis [16]. The NUT language includes two parts: a specification language and a procedural language. We outline the specification language here and present a small example.

\subsection{Specification Language}

There are built-in classes for primitive data types: num, bool, text, prog, any. The last is a universal class, which should be narrowed to any well-defined $\overline{\text { class }}$ before a more detailed specification will be needed. A class is specified as a collection of declarations of the following form ( $\mathrm{C}$ is a class name):

Superclass declaration
super C;
- C is a superclass

Component declaration

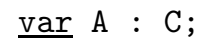

- A is a new component

which has a class $\mathrm{C}$

Method declaration

rel $\mathrm{R}$ : <axiom> <program>; - $\mathrm{R}$ is a new method

(the name $\mathrm{R}$ may be omitted.)

Equality declaration

$\underline{\text { rel }} \mathrm{A}=\mathrm{B}$;

- A and B are components with

the same value 
Equation declaration

$\underline{\text { rel }}$ e1 = e2;

- e1, e2 are arithmetic expressions which define a constraint on the variables occurring in them

A complete description of the NUT language can be found in [17].

\subsection{An Example}

A simple example of logical circuits description illustrates the usage of basic constructs of the language and program synthesis 14. We describe inverter, and-port and nand-port as classes of basic elements, and a logical circuit is described as a set of the interconnected basic elements.

\section{INVERTER}

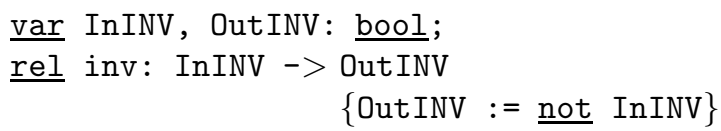

INVERTER has two boolean primitive type specifiers InINV and OutINV. The method inv specifies computations of OutINV from InINV which are performed by sequence of statements (body of the method) in the curly brackets. This method will be translated into the following axiom: InINV $\underset{\text { inv }}{\longrightarrow}$ OutINV where $i n v$ refers to the body of the method, i. e. to $\{$ OutINV $:=\underline{\text { not }}$ InINV $\}$.

AND

var InAND1, InAND2, OutAND: bool;

$\underline{\text { rel }}$ and: InAND1, InAND2->0utAND

\{OutAND:=InAND1 \& InAND2\}

NAND

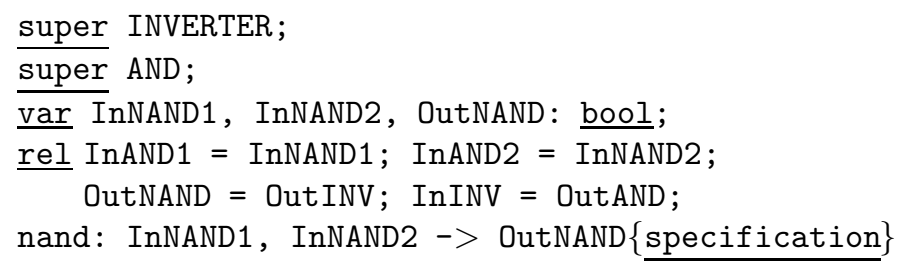

Let us follow the (automatic) translation of the class NAND into the set of axioms. Each equality is translated into two axioms, e.g. InAND1 $\underset{\mathrm{asg}}{\longrightarrow}$ InNAND1 and InNAND1 $\underset{a s g}{\longrightarrow} \operatorname{In} A N D 1$, where asg is the name of the standard function performing assignment. The keyword specification in the last method indicates that a function for the nand method should be synthesized automatically.

Other equalities and methods give the following set of axioms in the sequential form where and, inv and asg indicate methods which implement the corresponding formulae: 


$$
\begin{aligned}
& \vdash \text { InINV } \underset{\text { inv }}{\longrightarrow} \text { OutINV; } \quad \vdash \text { InAND1\&InAND2 } \underset{\text { and }}{\longrightarrow} \text { OutAND; }
\end{aligned}
$$

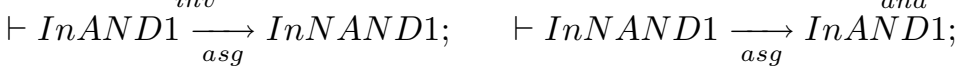

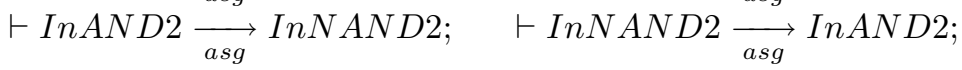

$$
\begin{aligned}
& \vdash \text { OutNAND } \underset{a s g}{\longrightarrow} \text { OutINV; } \vdash \text { OutINV } \underset{a s g}{\longrightarrow} \text { OutNAND; } \\
& \vdash \operatorname{InINV} \underset{a s g}{\longrightarrow} \text { OutAND; } \quad \vdash \text { OutAND } \underset{a s g}{\longrightarrow} \operatorname{InINV} \text {; }
\end{aligned}
$$

(Also axioms describing a class as a data structure which can be composed and decomposed are always introduced. We don't use them in the present example, and don't show them here.)

The theorem to be proven is $\vdash \operatorname{In} N A N D 1, \operatorname{In} N A N D 2 \rightarrow O u t N A N D$. Using the set of formulae and the inference rules of SSP $((\rightarrow-)$ - implication elimination and $(\rightarrow+)$ - implication introduction), the following proof can be made:

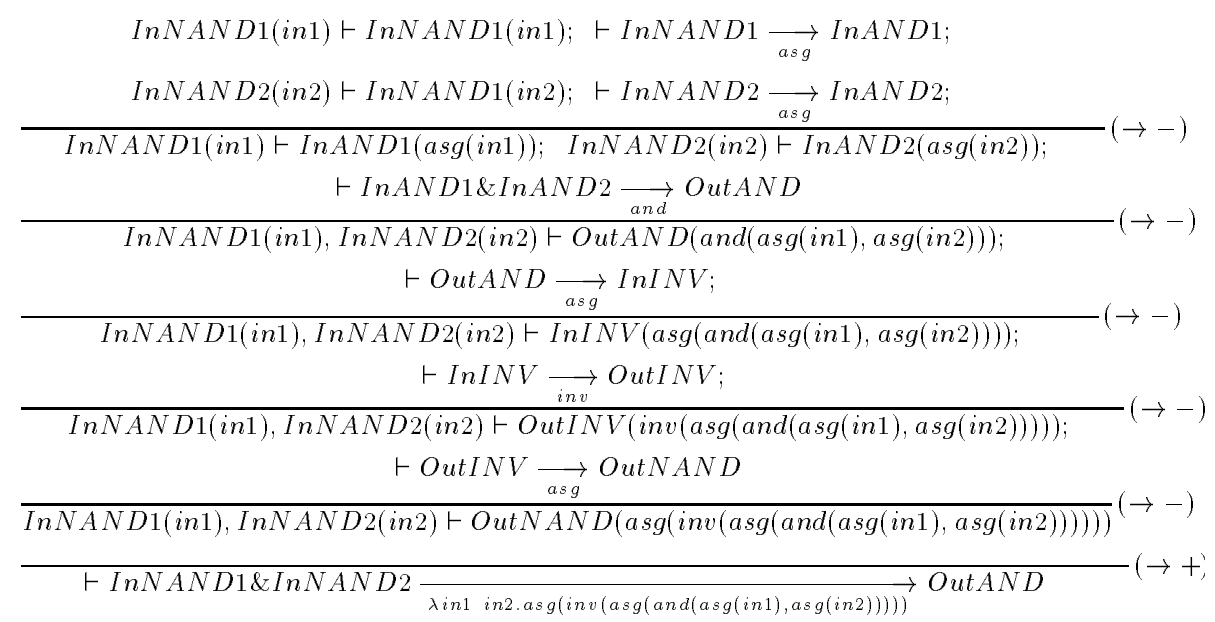

Q.E.D.

The extracted program is as follows

$$
\lambda i n 1 \operatorname{in} 2 . \operatorname{asg}(\operatorname{inv}(\operatorname{asg}(\operatorname{and}(\operatorname{asg}(\operatorname{in} 1), \operatorname{asg}(\operatorname{in} 2)))))
$$

or after optimization which removes assignments $(a s g)$ for equalities from the formulae

$$
\lambda i n 1 \operatorname{in} 2 . i n v(\operatorname{and}(i n 1, i n 2))
$$

The classes above can be used for description of different logical circuits. For example, the circuit from Fig. 11 can be specified by the following class in NUT. 


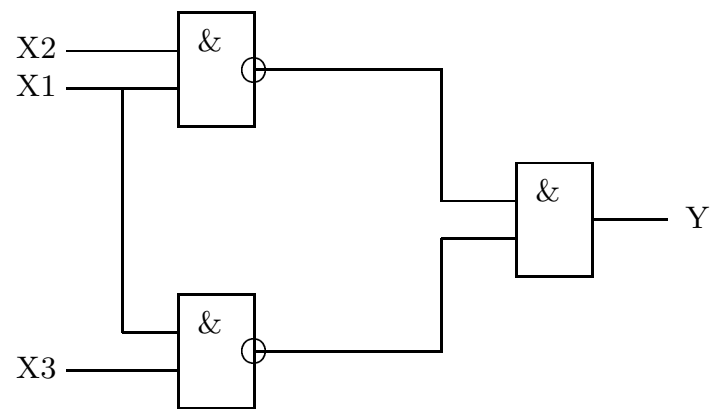

Fig. 1. A logical circuit

\section{SCHEMA}

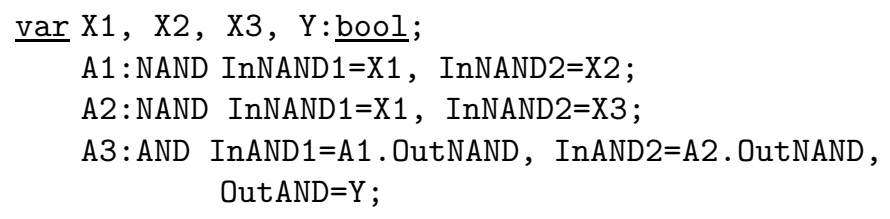

Some possible goals are as follows.

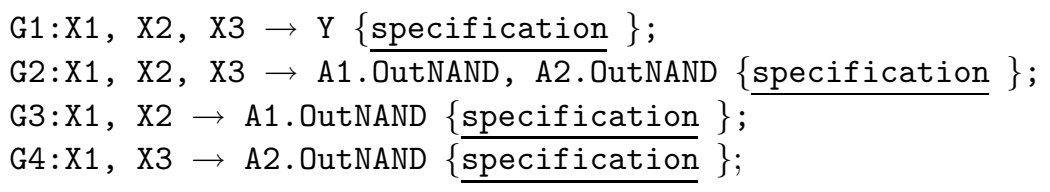

Programs for solving these goals are synthesized in a manner similar to the synthesis of the nand method.

This small example should give an idea how classes are used in specification. The approach is, however, scalable and the next sections demonstrate its application for specifications containing thousands of axioms and objects.

\section{Solving Numerical Problems in Mechanical Engineering}

This is a problem domain where the structural synthesis of programs has been in use for a long time, and applications are in the form of numerous packages. Fig. 2 (taken with permission from [8]) shows a set of packages and their interrelations in mechanical design which have been developed or are under the development with the SSP tools. The largest is a package for calculating tear and wear of gears in gearboxes, used during several years in the Tallinn Excavators Factory.

Mathematical models of typical machine parts: shafts, springs, housings etc. are traditionally presented as sets of algebraic equations and tabulated functions. Such a model is used in two different ways: either for checking the suitability 


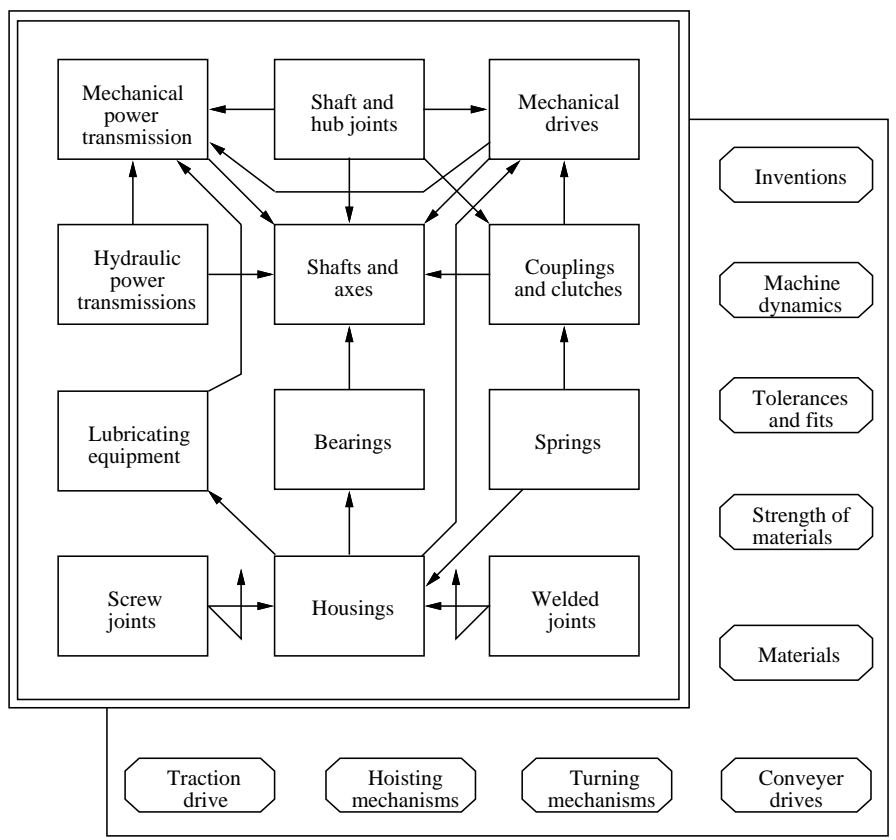

Fig. 2. CAD packages in mechanical engineering

of a given design by calculating the stresses and comparing them with their admissible values, or for calculating the dimensions of a device (machine part) from given design data. Traditionally, there are different programs for these two tasks. Applying the SSP, one can use one and the same mathematical model for both tasks, stating only different goals. Still, developing one universal model for a problem domain could be a difficult task. Object-orientedness and visual tools of the NUT system enable one to develop a set of concepts representing the ontology of a problem domain in such a detail that it becomes easy to specify each particular case of design in this ontology. We present here a simple example: development of a visual language for calculating loads and kinematics of gearboxes, introducing in this way also visual tools of the NUT system.

We start from the ontology for the problem domain. In the present case, the ontology consists of one concept: a gear (toothed wheel) and its features: diameter $D$, rotations per second $n$, linear speed $v$, torque $T$, tangential force $F$, module of teeth $m$ and number of teeth $z$. The concept of gear is specified as a class, and its features, which are numeric parameters in this case, become instance variables of the class. Fig. 3 3 shows a specification of the gear in a NUT class window. We can see the usage of equations in the class specification. An equation is always interpreted in NUT as a set of methods, one for every variable which can be computed from the equation. For instance, the equation

$$
\mathrm{D}=\mathrm{m} * \mathrm{z} \text {; }
$$


gives three methods whose axioms are the following:

$$
\begin{aligned}
& m, z \rightarrow D \\
& D, m \rightarrow z \\
& D, z \rightarrow m
\end{aligned}
$$

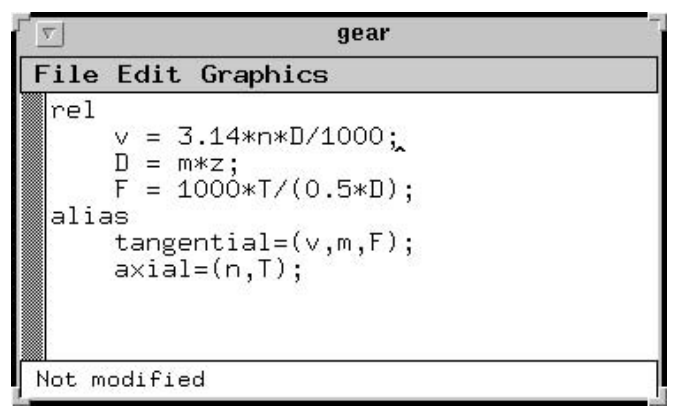

Fig. 3. Class of gear

There are two data structures tangential and axial specified in this class. These structures will be used for connecting gears tangentially and axially.

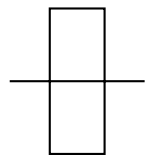

(a)

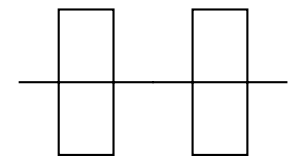

(b)

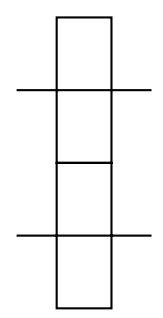

(c)

Fig. 4. Visual ontology of gears: (a) am image; (b) connected axially; (c) connected tangentially

Having specified the class gear, we associate an image with this class. This is done in a graphics image editor window opened from the Graphics menu of the class window visible in Fig. 3. The image of a gear, familiar to mechanical engineers, is simply a rectangle with an axis through it, Fig. 4, a. The ends of the axis should be defined as ports (graphical elements) by associating them with the name axial used in the class gear. These ports are for connecting gears axially as 
shown in Fig. 4 b. Connecting two images via ports means equality of of objects associated with ports. Looking at the class definition, we see that the axial connection of gears means equality of their torques and rotations per second. Also horizontal sides of the gear are defined as ports, for tangential connection in this case, Fig. 4.c. According to the specification, the tangential connection means equality of forces $F$, moduli $m$, and velocities $v$ of the connected gears. This gives us a visual language for specifying kinematics of gearboxes which is sufficient for solving computational problems about kinematics and loads in gearboxes.

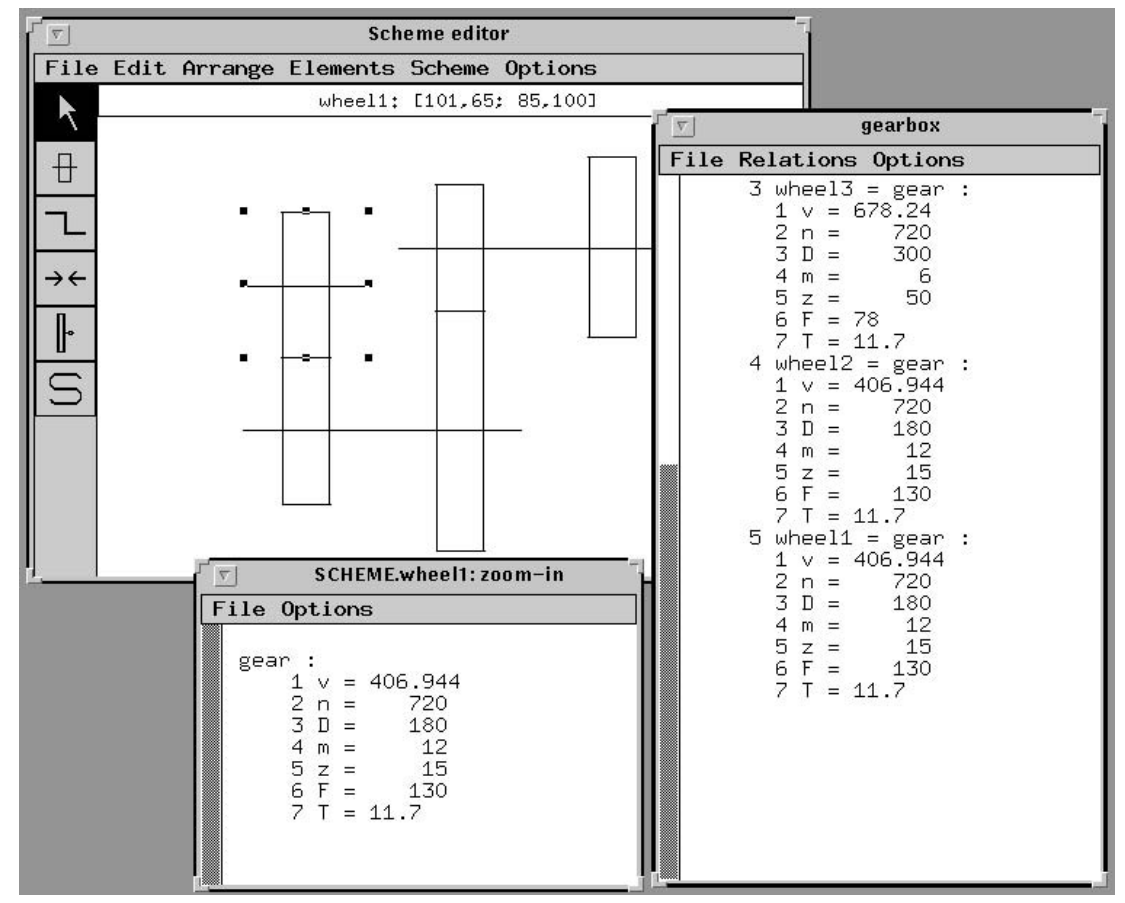

Fig. 5. Specification of a gearbox and results of computation

Fig. 5 shows a visual specification of a gearbox and results of some computations visualized for the gearbox and a gear in the NUTs built-in object windows. The example demonstrates how simple the development of a visual language can be for a restricted engineering domain, if a tradition of graphical representation of objects (gearboxes in this case) exists. This technique was used in building the packages for mechanical engineering. 


\section{Implementing Steam Boiler Control System}

In [1] an informal, but quite precise requirements specification of a boiler control system was presented as a benchmark for application of formal methods. The task was to develop a provably correct implementation of the control system. We solved the task by using the NUT language for encoding the refined requirements and synthesizing programs from these specifications [2]. The automatic synthesis guarantees correctness of the programs with respect to the requirements.

The control program consists of two parts: SysModel - simulator for the prediction of the behavior of the physical system, and Controler - control algorithm implementation. Besides that, another system simulator was developed which operated instead of the actual boiler during the testing and demonstration phases. Considerable part of the latter was identical to the SysModel. The structural synthesis of programs was used in different ways when implementing the simulator and the control algorithm as we shall describe here. The SysModel simulates the behavior of the steam-boiler system as a whole, partly on the basis of the information obtained from the measuring instruments, and partly by reflecting the actions performed by the Controler. It compares also the measured steam flow and water level with their predicted values for detecting the failure of level and steam measuring units. It delivers the adjusted values to the Controler for making the decisions. The SysModel is specified visually on the top level by using the scheme editor as shown in Fig. 6.

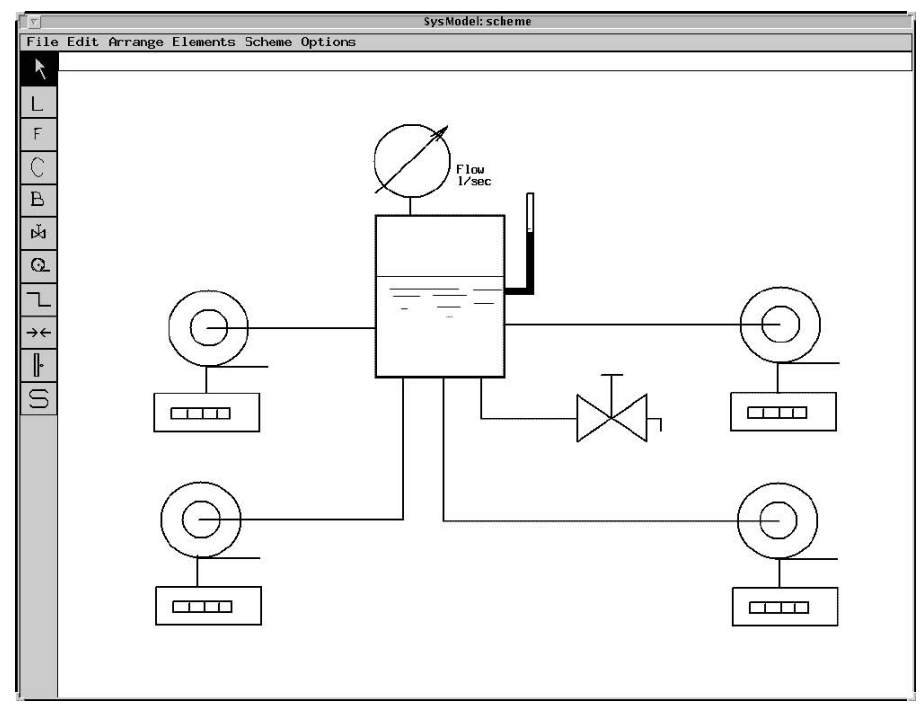

Fig. 6. Visual specification of SysModel of boiler system

Components of the SysModel represent physical units and are specified as subclasses of the class Device: 
class Device

var

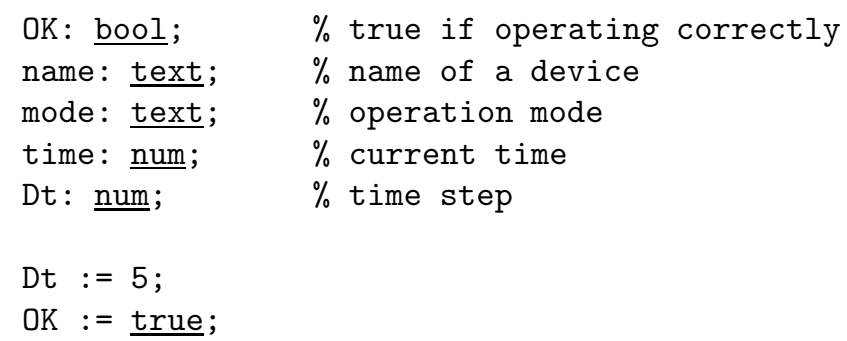

In particular, class Boiler is a subclass of Device and BoilerParameters, and represents the configuration of the boiler environment, including a valve and pumps:

class Boiler

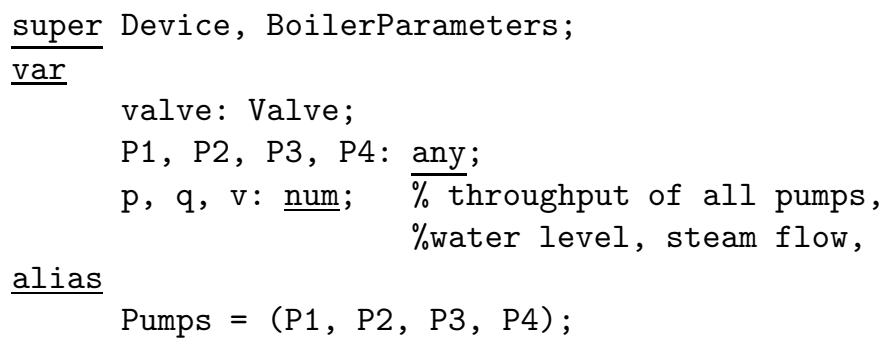

Class BoilerModel is a subclass of Boiler, it represents the actual mathematical model of the boiler, and includes numerous equations in the same form as written in the requirements specification [1]. It is a complete specification of boiler as a physical device suitable for synthesis of programs for finding different sets of values of parameters (raw, calculated, adjusted).

Another part of the control system - the Controler was implemented in a different way. It was designed as a production system. Rules given in the initial requirements specification were represented as methods of the class ControlerRules. Axioms served as guards of the rules. A goal for synthesis fired the rules which satisfied the firing conditions. Fairness of the set of rules had to be verified manually after writing all the rules.

\section{Analysis of Hydraulic Systems}

This is a problem domain where structural synthesis has been used for solving a number of large problems. We describe here two of them. Several years ago G. Grossschmidt introduced a multi-pole method of description of models of elements and subsystems of hydraulic systems [7. This enabled him to develop a rich set of models of components of hydraulics which appeared well suited for implementation in the visual environment provided by NUT, and has been 
used in simulation of hydraulic systems of aircrafts, cars and machine tools. The paper [6] describes analysis of statics and dynamics of an airplane aileron position control - a work done under a contract with the Antonov Airplane Plant in Kiev, Ukraine. The position control mechanism of ailerons is an electro-hydraulic servo drive. Because of the extremely high requirements for the static, steady-state and dynamic characteristics of the control system, a detailed simulation is required. The model includes a multitude of nonlinear and changing relations with many loops. Analysis of static and steady-state characteristics is done in principle in the way as we described for gears. The difference lies in the complexity of models which does not cause practical difficulties. The NUT synthesizer can handle tens of thousands of variables and thousands of axioms which is sufficient for solving the present problem.

Analysis of dynamics is performed by means of simulation of the mechanism described as a network of interconnected components in the NUT scheme editor window. The main simulation procedure is implemented as a method of a process class used as a superclass of the whole control system model. As the process class is used in many applications, we describe its simplified version here.

class process

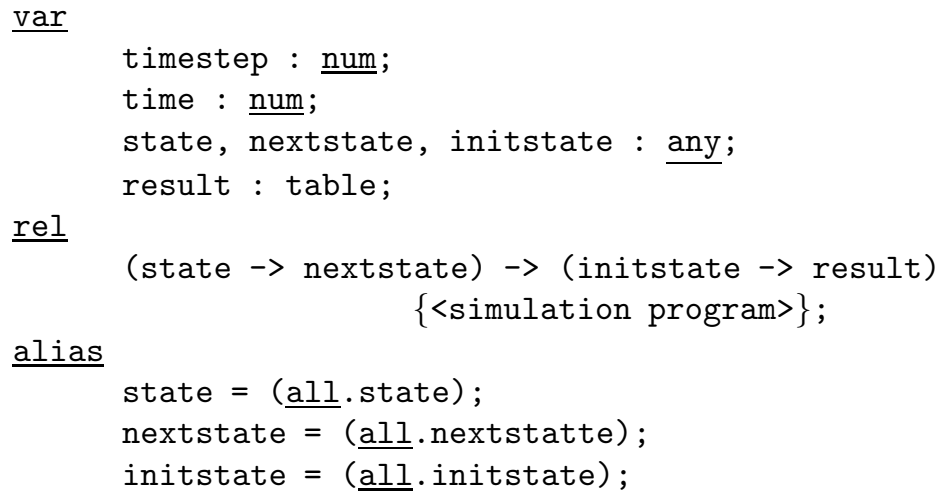

The alias statements in NUT define state, nextstate and initstate as structures (vectors) of variables with same names taken from components of the model. The axiom

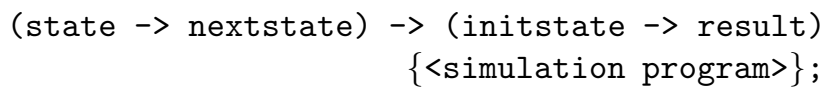

states that the result is computable from the given initstate as soon as an algorithm can be synthesized for computing nextstate from the given state. The simulation program is a loop over given time with the given timestep, computing nextstate and substituting nextstep for the step at each repetition.

Another problem concerns a load-sensitive hydraulic drive shown in Fig. 7. The functional elements of the drive are actuator AC, hydraulic cylinder HC, check valve $\mathrm{CHV}$, electro-hydraulic proportion valve EHPV, elasticity EL, flow 


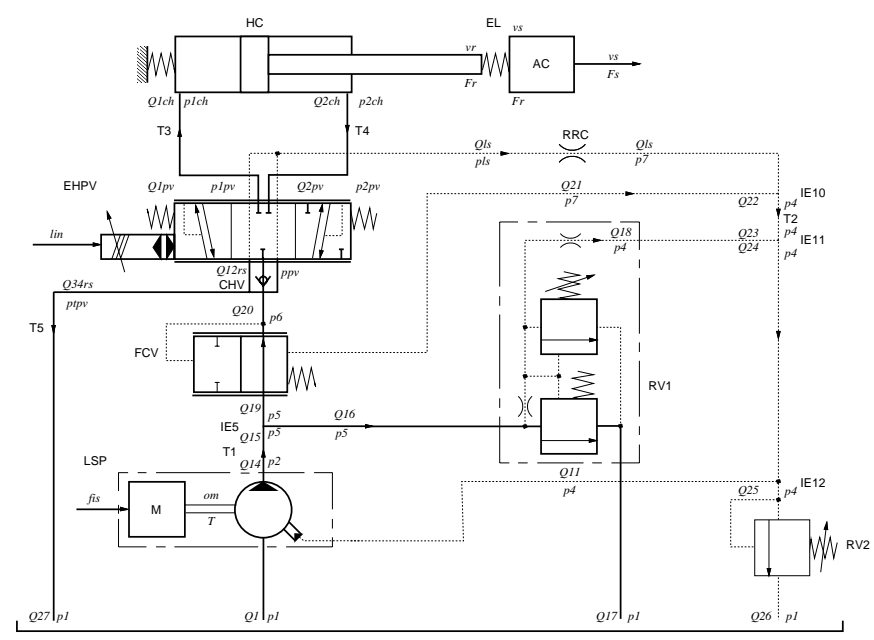

Fig. 7. Load-sensing pump

control valve FCV, interface elements (tee couplings) IE1,..., IE12, load-sensing pump LSP, driving motor M, hydraulic resistance RRC, relief valves RV1 and RV2, tubes $\mathrm{T} 1, \ldots, \mathrm{T} 5$. This problem concerns a stiff dynamic system, i.e. a system where both high and low frequencies are essential for obtaining a correct solution. This complicates the simulation task - one has to perform a large number of simulation steps in order to obtain required precision for the high frequencies, and to cover sufficiently long time for the slower part of the system. The total number of objects involved only in simulation of the hydraulic cylinder with tubes is already more than 50000 .

\section{Design and Analysis of Radar Coverage}

Modeling of a surveillance radar coverage (see an example of output of this package in Fig. 8), performed under the contracts with Estonian Ministry of Defense and Estonian National Maritime Board is an example where the user interface with volatile graphical objects has been useful. It means that graphical elements drawn from the program can be kept connected to instance variables of the object referred to in the drawing command. As a consequence, manual manipulation, (moving, reshaping etc.) of the image causes corresponding changes of the connected object. For instance, when calculations show that the radar cannot reach a certain point from a hill because of the shadow of a large object, one can find a new position for the radar by moving the image of radar (the dot in the center of the coverage picture) to another point and introduce new coordinates before the next iteration of the modeling.

The package of radar coverage analysis interoperates with the digital map including information about types of earth ground, buildings and their reflection properties. A specification of the radar coverage model uses classes of radars, i.e. 
sets of equations and parameters that describe characteristics of certain radar device and its properties of propagation of electromagnetic radiation (see details in [3]). There are also classes that describe attenuation of electromagnetic energy in atmosphere and models for different meteorological conditions such as rain, fog and clouds that can affect to the propagation of radar waves. In particular, we were interested in radar waves propagation when coastal area should be watched. Very different models of clutter must be used and switched on/off when the radar turns its beam from a water surface to land and back. This is an example of a real application of the system where hundreds and thousands of objects and geographical constraints must be taken into account. The program synthesis used provides correct computations of new surveillance state after changes in the specification of the model caused by moving radar positions or declaring changes in weather conditions, for instance.

To achieve the appropriate efficiency, the package has been implemented on the basis of the distributed NUT system [22] built upon PVM software. General control structure of the modeling task is represented as a method with subtasks (see section 2) where a subtask specifies a computation of the coverage by one radar. The distributed NUT assigns automatically these subtasks to available computers and spawns corresponding processes. The planning and computation of the coverage of different radars is performed in parallel and the results are returned to main process that visualizes integrated coverage as shown by example in Fig. 8

Some administrative and domain-specific control structures useful for development of parallel NUT programs were described in [22]. In our case, there is quite little attention to be paid to synchronization as radars do not communicate while working. For efficiency reasons, we have to deal carefully with resources management here. The program synthesis turned to be useful also for balancing the work load of workstations for parallel computing.

\section{Simulation of Mobile Communication}

The problem to be considered is analysis of accessibility for sites of wireless communication networks [1]. This example demonstrates all specification facilities of the NUT system. The main blocks are station and client which are basic nodes of a network to be modeled. Here we consider a GSM type network where objects of class Station refer to base stations i.e. non-movable radio transmission centers that have channels where clients can take a connection. Another class - Client (e.g. mobile phone) is a moving transmitter that may try to find free channel from a nearby base station. A client can use only one channel at a time, but while moving, the client has to change the base station or channel every time when transmission quality is not good enough to keep existing connection.

We assume using the dynamic channel assignment algorithm [4] when defining classes of mobile communication environment. Besides the classes Station and $\mathrm{Client}$ mentioned above, we need also a class Channel specifying 


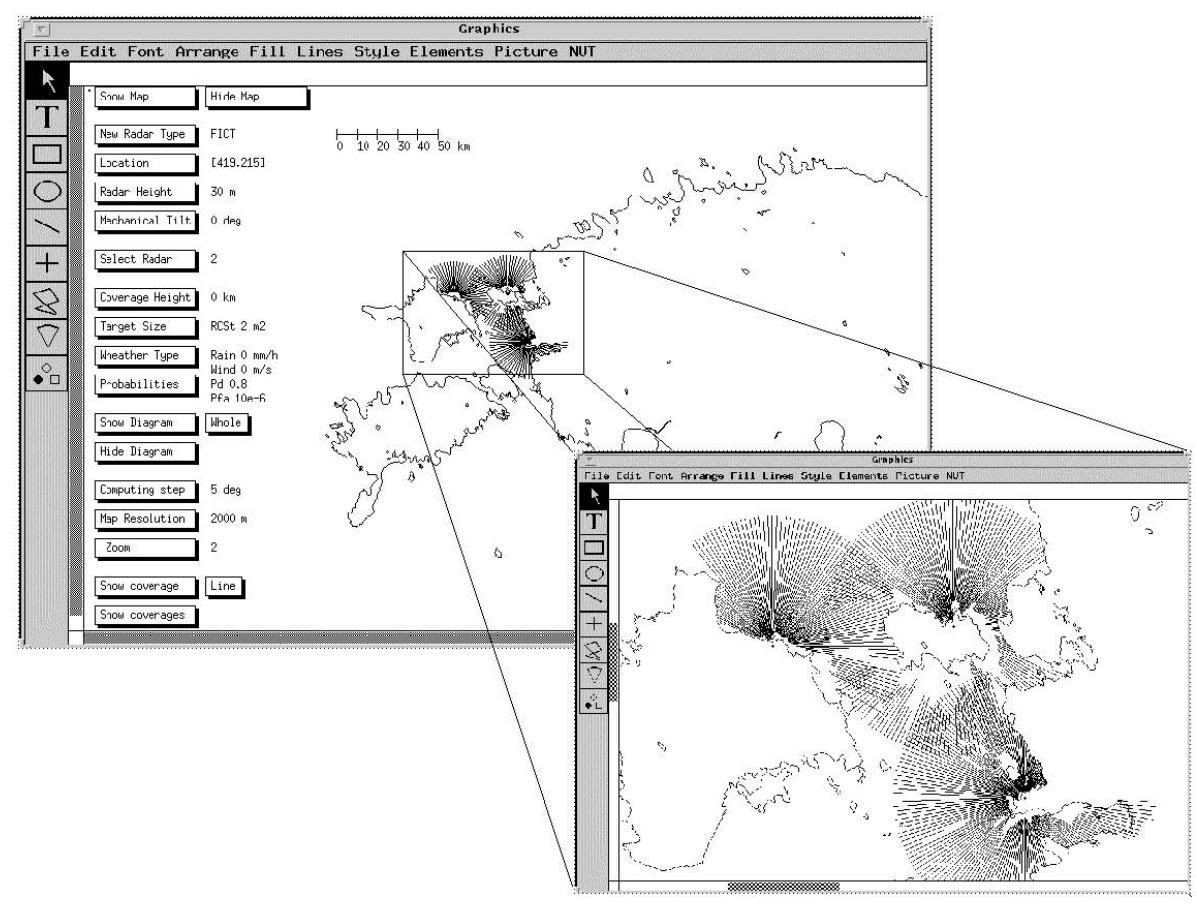

Fig. 8. Modeling of radar coverage

quality characteristics of the channel. There can be remarkable power losses in the channel caused by environmental conditions or long distance between sites connected. The noise in the channel created by other radio transmitters or coming from space is another reason why a client should lift transmission power or cancel connection. There are methods in the class Channel to compute these characteristics by request of clients or stations.

A client has the functionality to establish and keep connection to a base station nearby by choosing current channel in use and to achieve the best quality of connection using as little energy as possible. Clients check the beacon signals from base stations to decide from which station the received signal is highest and negotiate with the base stations assigned to them about characteristics and availability of channels. This functionality is implemented by the class Client.

The class Station specifies computations that a base stations has to perform for replying requests from clients as well as establishing of paths between clients that want to communicate.

To simulate a mobile network with respect to its real-time behavior, interactive graphics becomes useful. Here we specify base stations and clients in a scheme editor window. Images of clients and stations have a point whose coordinates are passed to the object and can be used for further computations. Stations and clients have the method Locate for visualizing their actual posi- 
tion and state in the specific graphical output window (upper right window in Fig. 9). This method can be activated by clicking on an element in the scheme editor window.

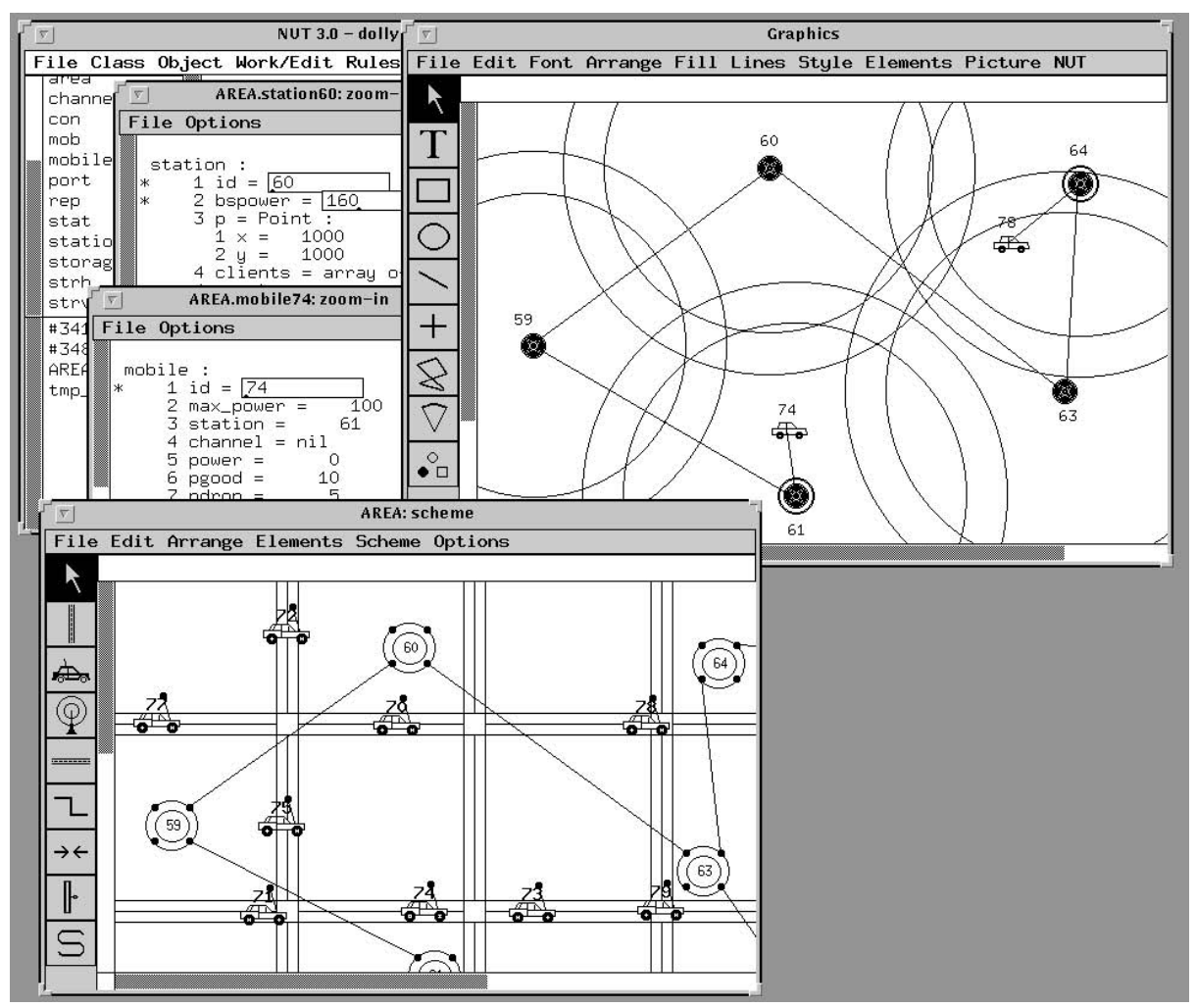

Fig. 9. Simulation of wireless communication (antennas and cars present base stations and clients/mobiles respectively)

The system allows to specify processes in the scheme editor window (moving a car ahead by a specified distance and/or changing radiation conditions, for example) that may influence the reliability of channels in use. Program synthesis is used for construction of algorithms that provide visualization of new situation in the environment and to compute the corresponding new communication paths between sites. Traditional routing algorithms can be used for modeling of path establishing processes. A specialization of the planning mechanism of NUT system to get a program for path search in mobiles network has been discussed but not implemented yet. 


\section{Concluding Remarks}

Applications of the structural synthesis of programs presented here are selected from various problem domains bearing in mind their practical usefulness and credibility. They all have been presented already as technical reports or even journal papers. However, with exception of the boiler control problem, they are written from the point of view of a particular application domain. For instance, the papers on the analysis of hydraulic systems [7, 6] give detailed description of models of hydraulic components and results of the analysis, but mention the software aspect only briefly. The boiler control example has been included into the present paper as a solution of a well-known benchmark. Our aim in selecting just these application examples has been to demonstrate the practical applicability of completely automatic program synthesis in problem domains, varying from simple engineering calculations to simulation of complex dynamic systems and parallel computing on a set of workstations. The fact that we use structural synthesis of programs has no principal importance. The main message of our presentation is that the deductive synthesis of program is sufficiently mature for automatic usage in software practice. One important lesson learned from our applications is that the automatic synthesis of programs should be supported by conventional software development tools: visual editor, object oriented programming language for implementing preprogrammed parts of the software etc. This seems to be true for any automatic application of formal methods. One can say it in another way: the synthesis is only one of the features of software development environments. It should be available for applications where it can be useful, and the amount of synthesis used may vary largely from one application to another. In our case, the synthesis appeared useful for developing visual specification languages, simulation software, and highly interactive computing even in the case of considerable amount of number-crunching.

\section{Acknowledgements}

This work was partially financed by Andersen Consulting Research Foundation and Estonian Science Foundation (grant \#2238). We would like to thank M. Addibpour, A. Kalja, T. Tiidemann, J. Pahapill, G. Grossschmidt and V. Kotkas for their kind support with information.

\section{References}

[1] Abrial, J.-R., Börger, E., Langemaack, H.: The Stem-Boiler Control Specification Problem. A Problem Suggestion for the Dagstuhl Meeting on Methods for Semantics and Specification, Lecture Notes Computer Science Vol. 1165, SpringerVerlag, Berlin Heidelberg New York (1997)

[2] Addibpour, M., Tyugu, E.: Structural Synthesis of Programs from Refined User Requirements (Programming Boiler Control in NUT). Lecture Notes Computer Science Vol. 1165, Springer-Verlag, Berlin Heidelberg New York (1997) 
[3] Blake, L.V.: A Guide to Basic Pulse-Radar Maximum-Range Calculation. Part 1. Equations, Definitions, and Aids to Calculation. Naval Research Laboratory Report 6930, AS 701321 (1969)

[4] Chuang, J.: Performance Issues and Algorithms for Dynamic Channel Assignment. IEEE Journal on Selected Areas in Communications, 11, 6 (1993) 955-963

[5] Coquand, T., Nordström, B., Smith, J.M.: Type Theory and Programming, Bulletin of the EATCS, Vol. 52 (1994) 203-228

[6] Grossschmidt, G., Pahapill, J.: Computing the Statics and Dynamics of Airplane Aileron Position Control Using the NUT Language. Proc. Estonian Acad. Sci. Engineering, 1, 1 (1995) 32-50

[7] Grossschmidt, G., Vanaveski, J.: Composing Multi-Pole-Model Block Schemes for a Load-Sensing Hydraulic Drive. Proc. Estonian Acad. Sci. Engineering, 3, 4 (1998) 209-224

[8] Kalja, A., Tiidemann, T.: A Conceptual Framework for Software Developing in Mechanical CAD. In Leinonen, T. (ed.): Proc. of OST-97 Symposium on Machine Design, Acta Univ. Oulu, C 109 (1997) 120 - 128

[9] Kalja, A., Tiidemann, T.: Supporting Mechanical Design with Visual Programming in the NUT Environment. In Leinonen, T. (ed.): Proc. of OST-98 Symposium on Machine Design, Acta Univ. Oulu, C 130 (1998) 163 -172

[10] Kotkas, V.: Intelligent Software Tools for Data Communication Modeling. In: László Varga (ed.): Proc. of the Fourth Symposium on Programming Languages and Software Tools, Department of General Computer Science, Eötvös Loránd University, Budapest (1995) 279 - 287

[11] Kotkas, V., Harf, M., Lõugas, R., Kann, E.: Positions of Coastal Radars Around Tallinn Bay. Final Report on the Contract, Institute of Cybernetics, Tallinn, (1998) - in Estonian

[12] Lowry, M., Philpot, A., Pressburger, T., Underwood, I.: Amphion: Automatic Programming for Scientific Subroutine Libraries. In Proc. 8th Int. Symp. on Methodologies for Intelligent Systems, Lecture Notes Computer Science Vol. 869, Springer-Verlag, Berlin Heidelberg New York (1994) 326-335

[13] Lowry, M., van Baalen, J.: Meta-Amphion: Synthesis of Efficient Domain-Specific Program Synthesis Systems. Automated Software Engineering, 4 (1997) 199-241

[14] Matskin, M., Komorowski, J.: Partial Structural Synthesis of Programs. Fundamenta Informaticae 31 (1997) 125-144

[15] Mints, G.: Propositional Logic Programming. In: J. Hayes et al. (eds.): Machine Intelligence, Vol. 12, Clarendon Press, Oxford (1991) 17 - 37

[16] Tyugu, E.: Using Classes as Specifications for Automatic Construction of Programs in the NUT System. Automated Software Engineering, 1, (1994) 315 - 334

[17] Uustalu, T., Kopra, U., Kotkas, V., Matskin, M., Tyugu, E.: The NUT Language Report. The Royal Institute of Technology (KTH), TRITA-IT R 94:14 (1994)

[18] Uustalu, T., Tyugu, E.: Higher-Order Functional Constraint Networks. In: Mayoh, B. et al.(eds.): Constraint Programming. NATO ASI Series F, Vol. 131, SpringerVerlag, Berlin Heidelberg New York (1994) 116 - 139

[19] Uuustalu, T.: Aspects of Structural Synthesis of Programs. The Royal Institute of Technology (KTH), TRITA-IT 95:09 (1995)

[20] Srinivas, Y.V., Jüllig, R.: Specware: Formal Support for Composing Software. In Proc. of the Conf. on Mathematics of Program Construction, Lecture Notes Computer Science Vol. 947, Springer-Verlag, Berlin Heidelberg New York (1995) 
[21] Stickel, M., Waldinger, R., Lowry, M., Pressburger, T., Underwood, I.: Deductive Composition of Astronomical Software from Subroutine Libraries. In: Bundy A., (ed.): Automated Deduction, Lecture Notes Computer Science Vol. 814, SpringerVerlag, Berlin Heidelberg New York (1994)

[22] Vlassov, V., Addibpour, M., Tyugu, E.: NUTS: a Distributed Object-Oriented Platform with High Level Communication Functions. Computers and Artificial Intelligence, 17, 4 (1998) 305-335 